\title{
Mesure de débit sur le terrain par ultrasons
}

\author{
S.E. Delattre, J. Biaggi \\ SAUR
}

Pour tout exploitant de réseau de distribution, la connaissance des volumes transités est évidemment capitale. Cette fonction est plus ou moins dévolue aux compteurs classiques, soit volumétriques, soit dits « de vitesse » (jet ou hélice axiale) avec pour objectif initial la facturation équitable de l'eau vendue ou achetée.

La nécessité d'une gestion plus stricte conduit à la connaissance non plus de volumes, mais de débits instantanés, non plus en extrémités de réseau, mais dans les conduites elles-mêmes. On peut déplorer que même pour des réseaux entièrement neufs, on ait omis d'installer des appareils de mesure de débit ou de volume le long des conduites principales. Bien entendu, de tels appareils n'existent pas non plus sur les réseaux anciens et il aura fallu de puissants impératifs économiques pour en installer sur le réseau parisien. Donc, dans la plupart des cas, l'exploitant est amené à utiliser des appareils portables que l'on place où l'on peut sans pratiquement jamais pouvoir respecter la symétrie axiale du champ des vitesses, symétrie requise pour les débitmètres existants.

Nous vous proposons de relater quelques-unes de nos expériences les plus marquantes de tentative de mesure de débit sur le terrain, dans des conditions hydrauliques tout à fait déconseillées par les fabricants d'appareils, tout en nous limitant au cas des mesures par ultrasons qui pour l'instant sont les plus faciles d'emploi, en utilisant des sondes non intrusives. Nous avons cependant une expérience intéressante avec des manchettes à sondes mouillées dont nous parlerons plus loin.

\section{Les sites de mesure et leurs difficultés}

On peut, pour clarifier l'exposé, distinguer 4 types de sites :

\subsection{Station de pompage}

L'objectif de la mesure peut être de retracer les caractéristiques des pompes de la station dont l'âge laisse présumer une chute de performances et surtout une disparité entre les différentes pompes avec tous les dangers que présente alors la marche en parallèle.

On peut aussi, dans bien des cas se contenter de connaître à un instant donné le débit injecté dans le réseau, surtout dans le cas des réseaux surpressés afin d'avoir une idée de la consommation instantanée et de son évolution au cours de la journée.

Il est, en général, impossible de fixer les sondes sur une conduite d'aspiration de pompe, faute de place, de même sur le tronçon de refoulement. Reste le collecteur de refoulement souvent équipé d'un compteur plus ou moins fiable, souvent d'un diamètre identique à celui de la conduite de refoulement.

On se trouvera donc confronté surtout aux difficultés de trouver une longueur droite, aux perturbations dues au compteur et enfin aux basses vitesses quand ne tourne qu'une seule pompe.

\subsection{Regard de réseau}

Il s'agit le plus souvent de regards exigus construits à l'économie abritant, soit des vannes, soit une ventouse. Il peut cependant arriver qu'on trouve une petite longueur droite pour poser les sondes sur une même génératrice, ce qui représente 2,5 à 3 diamètres.

A condition que l'écoulement ne soit pas perturbé par la vanne, les difficultés de la mesure proviennent de la méconnaissance du diamètre intérieur exact et des trop basses vitesses fréquemment rencontrées au moment de la mesure, les canalisations étant dimensionnées pour le jour de pointe et l'heure de pointe.

\section{Flow measurement on site with ultrasonic devices}

Instantaneous flowrates measurements in water distribution networks are required for improving network operation and water quality control in the mains. The ultrasonic flowmeter with external probes is widely used and the best suited device for this function. Its accuracy is very high when operated in the design hydraulic conditions and provided that the pipe be clean and its geometry well known. In actual measurement conditions, however, many errors of various origins may occur and lead to unlikely, unreliable and unconsistent results. Nevertheless, data post-processing methods tailored to the specific purpose of a measurements campaign have been developed to identify, evaluate and correct these errors so as to provide useful results. 
On rencontrera les mêmes inconvénients si on se décide à ouvrir une tranchée pour mettre la conduite à nu. Dans ce cas, on est à peu près sûr de la symétrie du profil des vitesses mais encore faut-il que ces vitesses soient suffisantes, c'est-à-dire supérieures à $0,5 \mathrm{~m} / \mathrm{s}$. De plus, la fixation des sondes est souvent difficile faute de pouvoir passer une sangle sous la conduite; il ne faut pas oublier qu'on travaille dans l'eau, la boue, avec des cailloux coincés là où il ne faut pas, et la terre du talus de l'excavation qui a une tendance naturelle à regagner le fond de fouille. Il y a là un problème d'outillage mal apprécié par les fabricants de débitmètres.

\subsection{Poteaux incendie}

Sur les antennes de distribution, les poteaux d'incendie sont très pratiques, soit pour augmenter artificiellement la vitesse dans la conduite, soit pour déterminer la perte de charge dans un tronçon, à condition de pouvoir priver d'eau les abonnés qui sont raccordés entre les deux points de mesure de pression. Pour mesurer le débit qu'on impose alors à la conduite, en ouvrant le poteau, nous utilisons couramment un élément de canalisation neuve de $6 \mathrm{~m}$, prélevé sur le parc, ce qui permet de mesurer exactement son diamètre intérieur. Si le débit atteint effectivement les $60 \mathrm{~m}^{3} / \mathrm{h}$ réglementaires, on obtient avec un tube de $3^{\prime \prime}$ une vitesse de $3 \mathrm{~m} / \mathrm{s}$ suffisante pour obtenir un bon profil de vitesse et une bonne précision du débitmètre.

Les sondes externes sont placées à $4 \mathrm{~m}$ environ de la liaison avec le flexible de raccordement au poteau, donc à $2 \mathrm{~m}$ de l'extrémité qu'il y a intérêt à munir d'une vanne de réglage, ce qui permet d'ouvrir en grand la soupape du poteau. Les turbulences amont sont ainsi réduites et la contre-pression créée dans le tube de mesure évite toute remontée d'air par l'écoulement à gueule bée.

La précision obtenue peut être excellente si la pression dans l'antenne est suffisamment stable pour ne perturber, ni le débit, ni la mesure des pressions aux deux extrémités du tronçon d'antenne.

\subsection{Chambre de vannes des réservoirs et colonnes montantes}

Il fut une époque où les équipements de réservoirs étaient entièrement réalisés en fonte de sorte qu'une chambre de vannes arrivait à ne présenter aucune longueur droite compte tenu de l'encombrement des pièces de transformation, tés, coudes, vannes, etc...

Nous ne pouvons, dans ce cas, qu'utiliser les colonnes montantes qui malheureusement s'avèrent très souvent imperméables aux ultrasons. C'est bizarrement sur ces colonnes qu'on rencontre les échecs les plus fréquents sans pouvoir avancer d'hypothèse expliquant l'encrassement préférentiel dans ces types de conduites, même quand elles sont en acier.

Quand bien même on parvient à recueillir un écho acceptable sur le débitmètre, l'incertitude majeure demeure le diamètre intérieur surtout sur les vieilles fontes. L'appareil mesureur d'épaisseur également à ultrasons donne en fonction du point d'application des écarts de un à trois millimètres qu'on n'est pas sûr de pouvoir attribuer à des irrégularités d'épaisseur ou à des inclusions ou dépôts particulièrement denses offrant une certaine conductibilité aux ultrasons.

\section{Exemples}

\subsection{Essais avec sondes externes}

A part ces échecs qui représentent 10 à $15 \%$ de nos tentatives de mesure de débit qui sont au nombre d'environ une centaine par an, nous avons obtenu des valeurs avec un écho correct, mais que valent-elles ? Dans les conditions hydrauliques douteuses, nous avons relevé sur une pompe entre 15 et $30 \%$ de plus sur le refoulement que sur l'aspiration, le zéro étant bien calé sur les deux extrémités utilisées en simultané. Quelle valeur faut-il prendre?

Sur une autre station, nous disposions, sur deux refoulements distincts, de compteurs Voltman récemment étalonnés par jaugeage d'une bâche dont la géométrie était parfaitement connue. Pour des débits très proches du point d'étalonnage, l'écart entre compteur et débitmètre était de $5 \%$, le débitmètre indiquant un débit inférieur au compteur. Il s'agissait d'une conduite de $300 \mathrm{~mm}$, les sondes externes à ultrasons étant placées à 8 diamètres en aval d'un coude à $90^{\circ}$, et le compteur à 10 diamètres, la vitesse étant de $5 \mathrm{~m} / \mathrm{s}$ environ et le zéro soigneusement calé.

Mais faut-il vraiment se fier aux compteurs car, pour l'un d'eux tout au moins, le coefficient de correction sur la lecture après étalonnage est passé de 0,934 à 0,981 en un an.

\subsection{Essais en sondes mouillées}

Avec l'objectif initial de mesurer la dérive d'un compteur au cours des ans, nous avons en sortie de réservoir réalisé une installation (fig. 1) comportant en série, sur chaque conduite, une manchette de mesure en inox pour sondes mouillées et un compteur. Il a été prévu en plus un piquage permettant l'utilisation d'un débitmètre électromagnétique à insertion. Dans un premier temps, il avait été placé un robinet-vanne à obturateur caoutchouté, juste en amont de la manchette, mais s'agissant d'une distribution d'eau potable, il n'a pas été possible de "faire le zéro ».

Le débitmètre à ultrasons comptait entre 7 et $8,5 \%$ de moins que le compteur, l'écart étant légèrement influencé par le débit. On a alors supposé que les vannes à opercule provoquaient, même en position ouverte, des turbulences qui faussaient la lecture du débitmètre à ultrasons. Elles furent donc supprimées et remplacées par des manchettes. L'écart monta à $13 \%$ pour une vitesse de $2 \mathrm{~m} / \mathrm{s}$ dans la manchette de mesure.

Nous avons alors fait faire une troisième manchette de mesure qui a été étalonnée au laboratoire de la SAGEP, en recherchant les valeurs de paramètre à introduire dans le logiciel du débitmètre pour qu'il indique exactement les valeurs de débit du laboratoire. L'écart réglé à zéro pour $1,9 \mathrm{~m} / \mathrm{s}$ atteignait $+2 \%$ à $0,75 \mathrm{~m} / \mathrm{s}$. La nouvelle manchette fut montée sur site et on reprit les mesures en paramétrant le débitmètre avec les mêmes valeurs qu'au laboratoire. Cette fois-ci, l'écart n'était plus que de $3 \%$, le compteur indiquant toujours plus que le débitmètre, tout au moins pour une vitesse de $1,35 \mathrm{~m} / \mathrm{s}$ en tenant compte de l'erreur de linéarité. On pouvait alors penser que le débitmètre était juste et que le compteur sur-comptait de $3 \%$.

Par contre, sur la manchette préexistante, non étalonnée, le débitmètre avec le même paramétrage qu'au laboratoire 


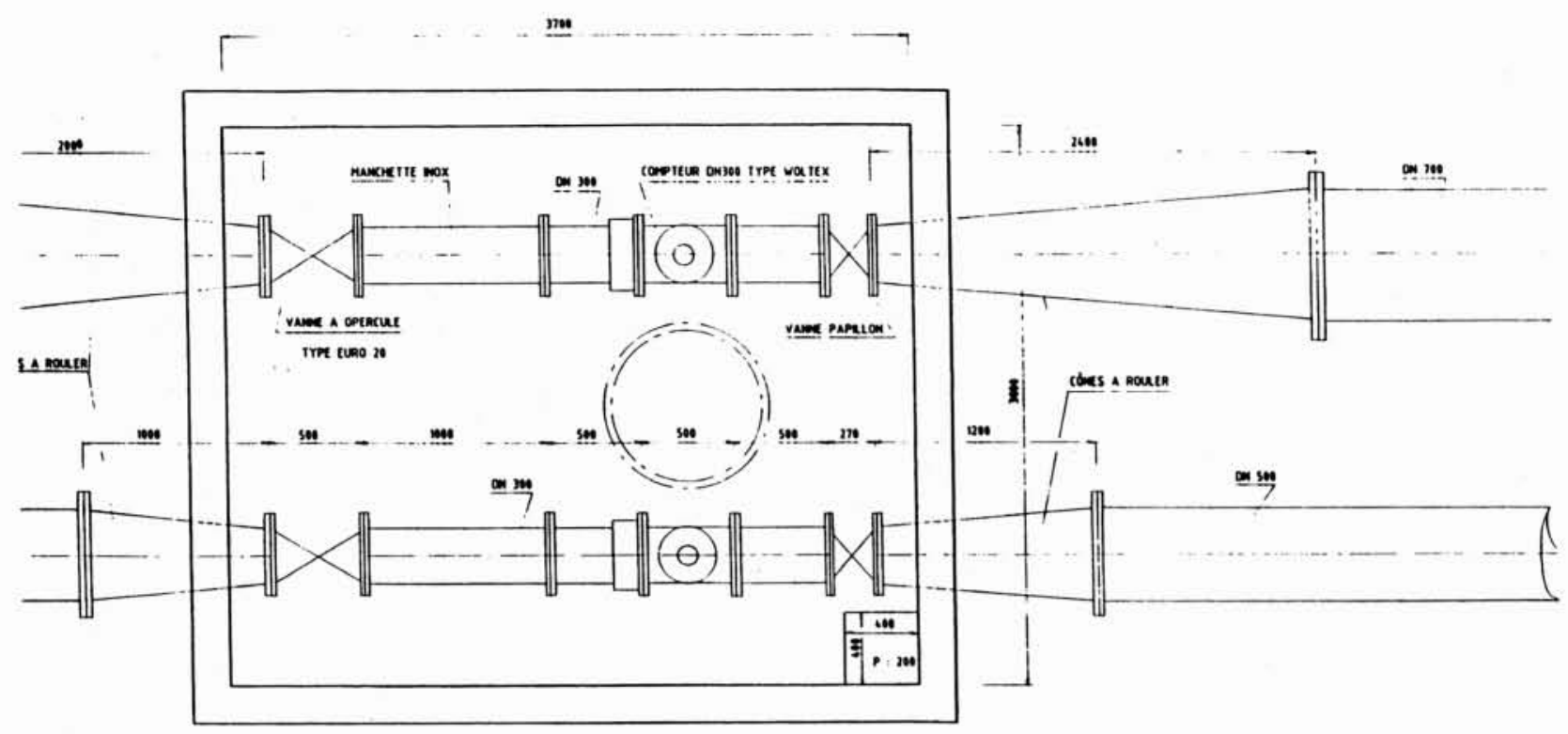

1. Installation pour essais en sondes mouillées.

sous-comptait de $10 \%$ par rapport au compteur. Inutile de dire que la géométrie des manchettes avait été contrôlée et en particulier le diamètre intérieur. L'ovalisation maximale était de $1 \mathrm{~mm}$, de sorte que la section de passage était connue à $0,5 \%$ près.

Enfin, on a procédé à des enregistrements par logger sur deux jours pleins mais en plaçant sur la dernière née des manchettes un débitmètre électromagnétique à insertion. Par rapport au compteur, le débitmètre à ultrasons indiquait $-10,5 \%$ et le débitmètre électromagnétique $+7 \%$ environ sur les volumes transités en 48 heures. Certes l'électromagnétique pouvait introduire des turbulences mais tout de même réduites pour les sondes à ultrasons puisque placé légèrement en aval.

Cela fait donc deux cas précis, l'un avec sondes externes sur conduite existante avec compteurs étalonnés, l'autre avec sondes mouillées montées sur manchette étalonnée, où les débitmètres indiquaient des valeurs inférieures aux compteurs.

Doit-on en conclure que, sur site, l'asymétrie du profil des vitesses incite les débitmètres à sous-compter, ou que les compteurs à hélices sur-comptent ?

\section{Les recoupements}

Nous nous sommes référés dans les exemples précédents à des compteurs. Bien évidemment, en laboratoire, on utilise des jauges mais pour mettre en évidence des erreurs possibles de linéarité, il faut maintenir un débit constant pendant la durée du remplissage et opérer sur plusieurs débits. Qu'il nous soit permis de mentionner, au passage, un résultat curieux obtenu au laboratoire de la SAGEP. Nous disposions d'une manchette de mesure en DN 150 (longueur $450 \mathrm{~mm}$ ) en mode reflex et sondes mouillées et sans but précis, nous avons monté, bridé à l'extrémité aval de la manchette un compteur axial, WEN, $Q N=150 \mathrm{~m}^{3} / \mathrm{h}$.
Nous avons obtenu avec le débitmètre une droite d'étalonnage jusqu'à $140 \mathrm{~m}^{3} / \mathrm{h}(v=2,20 \mathrm{~m} / \mathrm{s})$ puis au-delà, une lecture constante $140 \mathrm{~m}^{3} / \mathrm{h}$ alors que le débit croissait jusqu'à $200 \mathrm{~m}^{3} / \mathrm{h}$. Tout se passait comme si le débitmètre était saturé. Faute de temps, nous n'avons pu renouveler cette expérience dans d'autres conditions pour découvrir s'il s'agissait d'un phénomène systématique ou accidentel. Il serait de toute façon intéressant d'étudier l'influence des perturbateurs aval sur la précision de la mesure.

Sur le terrain, nous ne disposons pas de cuves jaugées mais il peut arriver que nous disposions de la courbe d'une pompe qui vient d'être essayée sur le banc du constructeur. Sachant mesurer les pressions avec une excellente précision de l'ordre de $0,5 \%$, on peut comparer le débit pompé déduit de la courbe avec celui qu'on peut mesurer au débitmètre.

Sur une station de pompage de reprise dans le Jura, nous avons ainsi retrouvé trois points qui ne différaient de la courbe que de $1 \%$, alors que le débitmètre à sondes externes était installé dans l'axe du refoulement à 2 diamètres en aval d'un clapet Clasar et juste avant la vanne d'arrêt, les vitesses étant de l'ordre de $2,5 \mathrm{~m} / \mathrm{s}$. Toutefois, nous avions pu mesurer sur une chute, le diamètre intérieur exact de la conduite et il faut bien le reconnaître, le clapet Clasar n'introduit pas de dissymétrie radiale du profil des vitesses.

Nous avons fait sur d'autres stations des mesures analogues sans jamais retrouver une aussi bonne précision, probablement en raison des coudes qu'on trouve presque toujours sur les refoulements.

A titre anecdotique, mentionnons une mesure faite sur un banc d'essai de constructeur de pompe, sur une conduite en acier DN 80 . Avec une vitesse de $10 \mathrm{~m} / \mathrm{s}$, l'écart des lectures entre le débitmètre à sondes externes et le débitmètre électromagnétique du banc, n'était pas décelable, bien que nous nous soyons installés à moins de 10 diamètres d'un coude. 
Il ressort de cette rapide exploration de nos expériences que les débitmètres à ultrasons sont capables du meilleur comme du pire.

\section{Méthodologie des mesures de débit en fonc- tion des objectifs}

Nous avons déjà évoqué les objectifs qu'un exploitant de réseau pouvait se fixer.

\subsection{Tracé des courbes de pompes}

La précision recherchée pour la mesure de débit va dépendre de la zone dans laquelle se trouve, sur la courbe $\mathrm{Q}, \mathrm{H}$ le point de mesure.

A gauche, dans la partie presque plate, on pourra admettre une erreur de $10 \%$ sur Q sans que la forme utile de la courbe soit sensiblement altérée. Par contre, à droite, dans la partie « pentue » on aimerait ne pas dépasser $\pm 2 \%$.

Cependant, le plus souvent, on ne parviendra pas à positionner les sondes dans une zone sans trop de turbulences. La seule solution à peu près valable est de se placer sur le collecteur de refoulement, quitte à ouvrir une tranchée et à manœuvrer des poteaux d'incendie, et faire passer le débit de la totalité des pompes disponibles moins une qu'on enclenchera et déclenchera à plusieurs reprises. On en déduira par différence au moins un point de fonctionnement de cette dernière pompe. En opérant par permutation, on obtient pour chaque pompe un point de fonctionnement que l'on comparera avec la courbe du constructeur.

L'intérêt de la méthode est évidemment de disposer dans la conduite d'une vitesse plus favorable à la mesure et de mettre en évidence les faiblesses de certaines pompes usagées quand elles fonctionnent en parallèle avec d'autres en meilleur état. De plus, en travaillant par différence des débits, on réduira sensiblement l'erreur relative par rapport à la mesure sur une seule pompe à la fois en éliminant pratiquement l'offset. En contrepartie, la méthode est longue surtout si l'on tient compte des contraintes d'exploitation.

La précision obtenue est très variable et difficile à préciser faute d'un étalon. Dans les cas favorables, on peut espérer $\pm 5 \%$.

\subsection{Mesure des pertes de charge}

Sur les réseaux, la difficulté essentielle est d'obtenir des débits suffisants, non seulement, pour le débitmètre mais aussi pour créer des pertes de charge significatives.

Sur les collecteurs, on ne peut espérer de mesures fiables à moins d'aménagements spéciaux tels que pose de manchette de mesure ou à la rigueur de colliers de prise en charge pour monter des sondes mouillées, et encore subsiste-t-il quelques doutes sur l'exactitude de la mesure comme nous l'avons vu.

Par contre, sur les antennes de distribution, on peut augmenter les vitesses en ouvrant un ou plusieurs poteaux d'incendie.

Un cas intéressant est celui pour lequel on peut isoler un tronçon entre deux vannes avec un poteau d'incendie à l'extrémité. On peut alors faire transiter dans le tronçon un débit de $60 \mathrm{~m}^{3} / \mathrm{h}$ ou même plus et obtenir si le diamètre est de l'ordre de $150 \mathrm{~mm}$, une perte de charge mesurable avec une bonne précision, le débit étant mesuré à la sortie du poteau par la méthode décrite plus haut. Il faut cependant que les abonnés acceptent une coupure d'eau, pendant au moins une heure et que le fontainier n'en oublie pas.

Dans tous les cas, on a besoin d'une très bonne précision, $2 \%$ étant le maximum acceptable, rarement atteint sauf en sortie de poteau d'incendie.

\subsection{Quantification des fuites}

Le prix des débitmètres à ultrasons condamne pratiquement leur utilisation pour la mesure systématique des petits débits, comme ceux que l'on rencontre en quantification de fuites quand on parvient à isoler un quartier. Il y a cependant une exception si on ne peut disposer du camion laboratoire avec son appareillage ; les débitmètres à ultrasons, ayant leurs propres batteries d'accumulateurs, peuvent être branchés sur un élément de conduite neuf en acier intercalé entre les deux flexibles reliant les deux poteaux d'incendie desservant le quartier. Un logger associé permettra de déterminer le débit minimum de nuit que l'on assimile habituellement au débit des fuites. Il conviendra cependant de mettre l'appareillage à l'abri du vol et du vandalisme et de bien choisir le diamètre de la conduite de mesure pour obtenir des vitesses significatives.

Dans ce cas précis, on n'a évidemment pas besoin d'une grande précision ; même en prenant la précaution d'attendre que la courbe du débit, en fonction du temps, soit suffisamment plate pour pouvoir prétendre qu'il s'agit bien d'un débit de fuite et non d'un consommateur nocturne, on ne peut séparer les fuites du réseau des fuites intérieures chez les abonnés sauf par approche statistique.

Les $2 \%$, voire même $5 \%$ d'erreur sur la mesure sont parfaitement acceptables pour décider s'il y a lieu d'entamer une campagne de recherche de fuites.

\subsection{Physiologie des réseaux}

Nous avons été amenés à modéliser une grande partie des réseaux urbains que nous exploitons. Cependant, faute de capteurs en nombre suffisant, il est difficile de suivre l'évolution des débits et pressions en temps réel aux points stratégiques. On est donc amené pour certains jours-type, à effectuer une campagne de mesure avec des appareils portatifs pour suivre l'évolution horaire du réseau et du même coup recaler le modèle mathématique établi initialement avec quelques valeurs moyennes et aussi des pertes de charge estimées.

On installe alors le plus grand nombre de débitmètres possible sur toutes les canalisations accessibles à la mesure et en particulier sur les colonnes d'arrivée et de départ des réservoirs, chaque débitmètre étant associé à un enregistreur numérique avec horloge.

En reportant les valeurs lues à chaque instant, dans le modèle mathématique, on obtient des bilans permettant de vérifier la cohérence des mesures, dont certaines seront éliminées et d'autres corrigées en fonction des critères habituels tels que vitesse résultante dans la conduite ou imprécisions dues aux turbulences ou encore mauvais état de la conduite.

L'erreur importante pouvant être faite sur les hypothèses de consommation, l'altimétrie du réseau, les pertes de 
charge de certains tronçons, une précision de $10 \%$ sur la mesure des débits reste acceptable et autorise le recours aux débitmètres à ultrasons dans des conditions très imparfaites.

Ce genre d'étude critique exige beaucoup d'expérience de la part des opérateurs qui auront toujours à estimer la précision, voire même la vraisemblance de telle ou telle mesure.

\section{Conclusion}

Les débitmètres portatifs à ultrasons sont très vite devenus intéressants pour les exploitants de réseau, mais dans leur état actuel sont incapables de corriger la principale source d'erreur représentée par les dissymétries de profils particulièrement graves à basse vitesse. Une amélioration est à espérer par l'utilisation de débitmètres multicordes qui, en sondes externes, permettront d'intégrer le profil sur deux diamètres orthogonaux.

L'inconvénient majeur pour l'utilisateur est la méconnaissance de l'erreur de mesure dès l'instant où l'appareil reconnaît un écho correct. Nous préférerions un appareil qui nous affiche un message tel que «mesure non valable " à un autre qui fournit une valeur incertaine.

Cependant, pour certains usages comme la quantification de fuites, la physiologie des réseaux et la mesure de perte de charge sur poteau incendie, la précision qu'ils assurent peut être de l'ordre de grandeur de celle recherchée. L'expérience et le jugement de l'opérateur sont alors primordiaux dans la validation de la mesure.

D'autres progrès seraient aussi souhaitables :

- un produit couplant adhésif qui évite de sangler les sondes,

- un intégrateur automatique fournissant le débit moyen sur un temps réglable afin d'éviter les chronométrages fastidieux et parfois douteux dans un environnement difficile. Il semble que les modèles les plus récents soient munis de ce perfectionnement.

A ce propos, il serait intéressant que des physiciens lèvent un doute sur l'existence réelle des oscillations de débit dans nos canalisations, lesquelles, sur pratiquement tous les types de débitmètres, atteignent jusqu'à $5 \%$ avec des périodes apparentes de l'ordre de 20 à 30 secondes. 